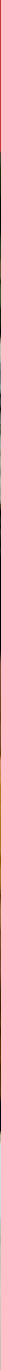

\title{
Impacts of a work sipport programme for youth on academic outcomes and employability in the Philippines
}

Helping young people to enter and remain in the workforce is considered critical for prosperous, stable and cohesive societies. However, youth unemployment remains a challenge for developing countries. In the Philippines, young people aged 15 and 24 comprise nearly half of all unemployed persons in the labour force. Finding work is hardest for those who do not graduate from high school.

In an effort to increase graduation rates among students and facilitate employment after schooling, the Philippines Department of Labor and Employment (DOLE) launched the Special Program for Employment of Students (SPES) in 1993. This programme, implemented by DOLE alongside provincial governments and municipal public employment service offices, links youth from low-income households (whether enrolled in school or not) to formal work opportunities with decent wages during school breaks. It is envisioned that the prospect of earning while at school will attract children to enrol in schools and improve retention among those already enrolled.

As part of the Philippines Evidence Programme, 3ie partnered with the National Economic and Development Authority to assist DOLE in commissioning an independent evaluation of SPES, a priority programme of the department.

To assess the effectiveness of SPES, 3ie supported researchers affiliated with Innovations for Poverty Action to conduct a process evaluation and a largescale randomised field experiment. This evaluation, conducted between 2015 and 2018, measured the programme's impact on participants' education, employability and employment outcomes.

\section{Highlights}

- The evaluation showed that SPES does not impact academic outcomes or work readiness in the medium term.

- SPES participation helped participants to enter the workforce by increasing their likelihood of being employed.

- To further enhance employment outcomes, there may be value in recasting SPES as a work programme, helping participants to build life skills in addition to providing better work opportunities.

- DOLE has acted upon some of the programmatic shortcomings highlighted by the evaluation. 


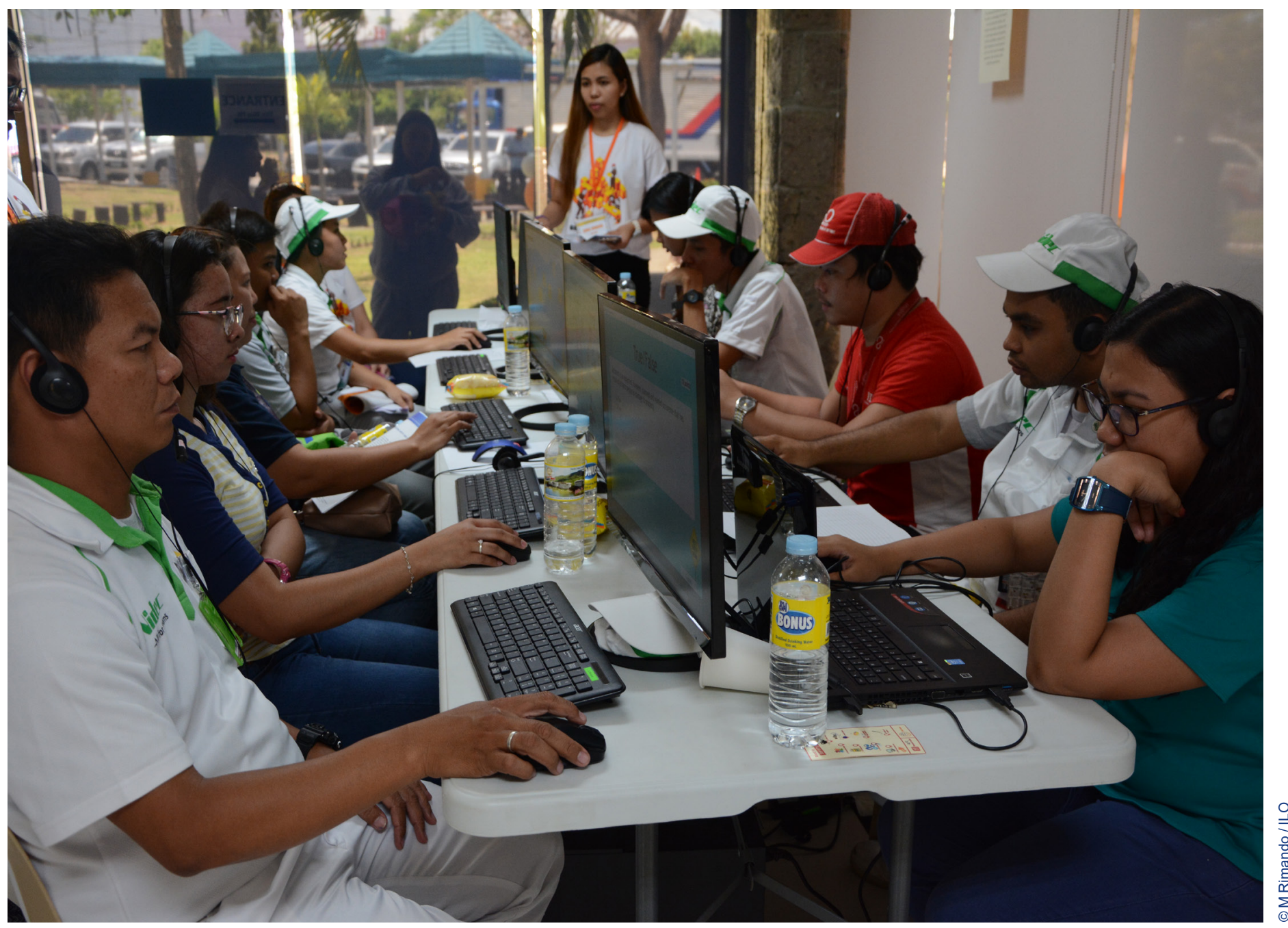

\section{Main findings}

The evaluation was implemented in five regions: the National Capital Region, Central Luzon (Region III), Western Visayas (Region VI), Central Visayas (Region VII) and Davao (Region XI).

In municipalities where summer programme batches were oversubscribed, a subset of applicants was randomly chosen to enrol for SPES, while the rest were assigned to a control group. In the final assignment, the treatment group consisted of 2,511 SPES participants and the control group included 1,285 participants. The two groups were compared 8 to 12 months later to understand the medium-term impacts of SPES participation on education and employment.

The researchers requested that 13 public employment service offices from each municipality with the highest enrolment in 2014 participate in the evaluation. However, they faced challenges in garnering local support for randomised acceptance into SPES, which stemmed from various political considerations, including 2016 being an election year. This led to several challenges, including a sample that was not representative of SPES participants and instances of non-compliance with randomisation. With these caveats, the researchers highlight the following findings:

- Education: SPES participation had no overall effect on school enrolment, graduation or grades. However, there was some evidence that the programme increases enrolment among males and students from relatively poorer families, who are at a higher risk of dropping out.

\section{- Work readiness and labour} market perceptions: SPES did not improve participants' selfesteem or self-reported life skills. Even when the programme improved participants' confidence about future work prospects, it did not change their expectation for better wages.

- Employment outcomes: At the time of the study, SPES participants had an increased likelihood of being employed in the private sector, a local government unit or a non-governmental organisation. The effects were larger for those enrolled in college.

n Targeting: The study found that men, high school students and students from economically poor backgrounds benefited more from SPES. This suggests that the programme's effectiveness can be improved by targeting such groups.

n Cost-effectiveness: Although the study showed promising impacts on employment outcomes for participating youth, the cost incurred by DOLE is prohibitive (over USD1,000 per job found). 


\section{How DOLE is using evaluation evidence}

Evaluation findings reinforced DOLE's view of recasting SPES as a work-support programme and adding a life skills training component. Following amendments to the SPES law in 2016, DOLE made changes to the programme to better target poorer participants, including an increase in the number of working days, from 52 to 78 , and expansion of wage subsidies to employers, from 40 to 60 per cent in low-income local-government units.

In recent years, DOLE has refocused the aims of SPES, with added emphasis on improving youth employability. The department is also working towards increasing privatesector participation for better on-the-job learning experiences. According to the study, a majority of current SPES participants are engaged in administrative tasks as part of their jobs in local government units.

\section{Lessons for future research}

Given the relatively short timeline and the limitations of this evaluation, it would be valuable to conduct a follow-up study to explore longer-term impacts of SPES, especially on educational outcomes.

Future research could also explore the consequences of SPES law amendments introduced in 2016.

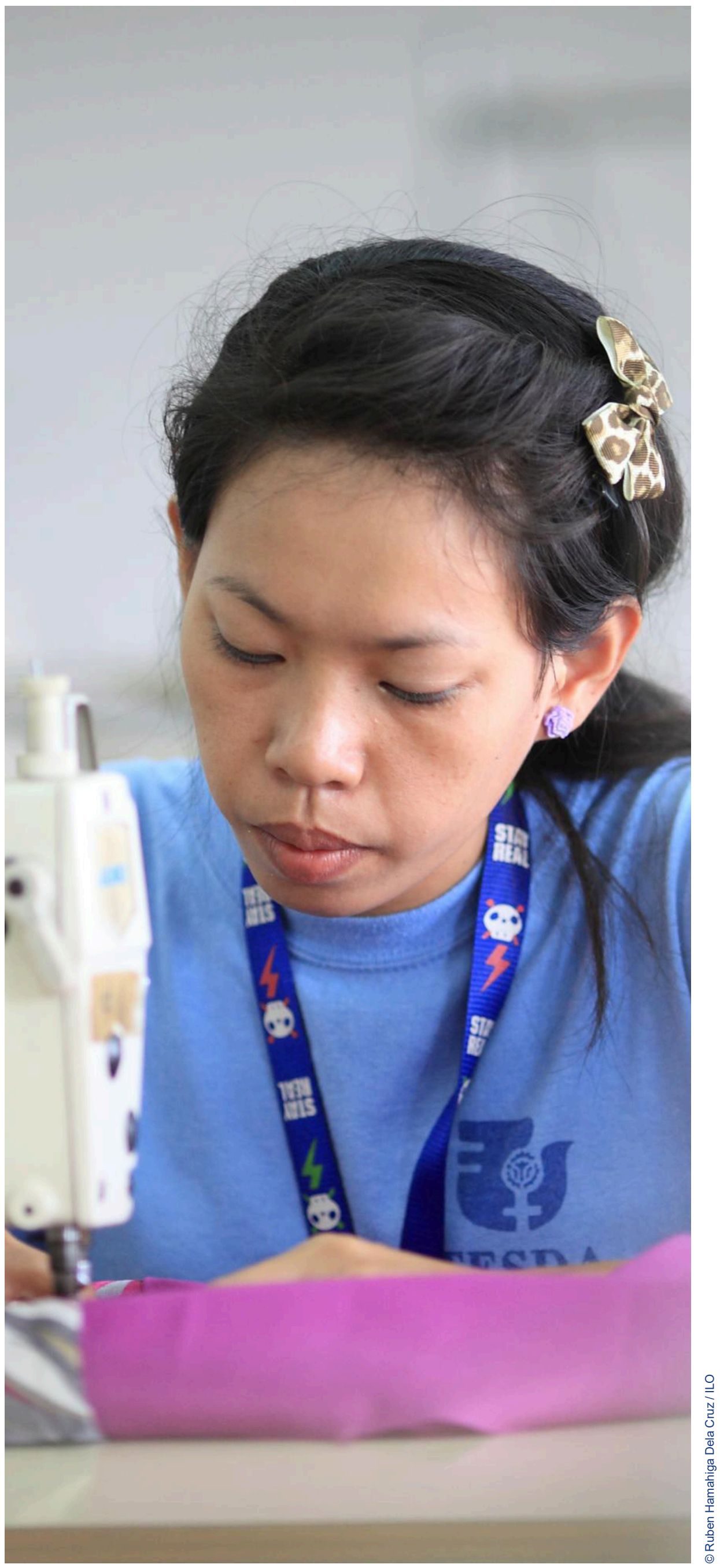




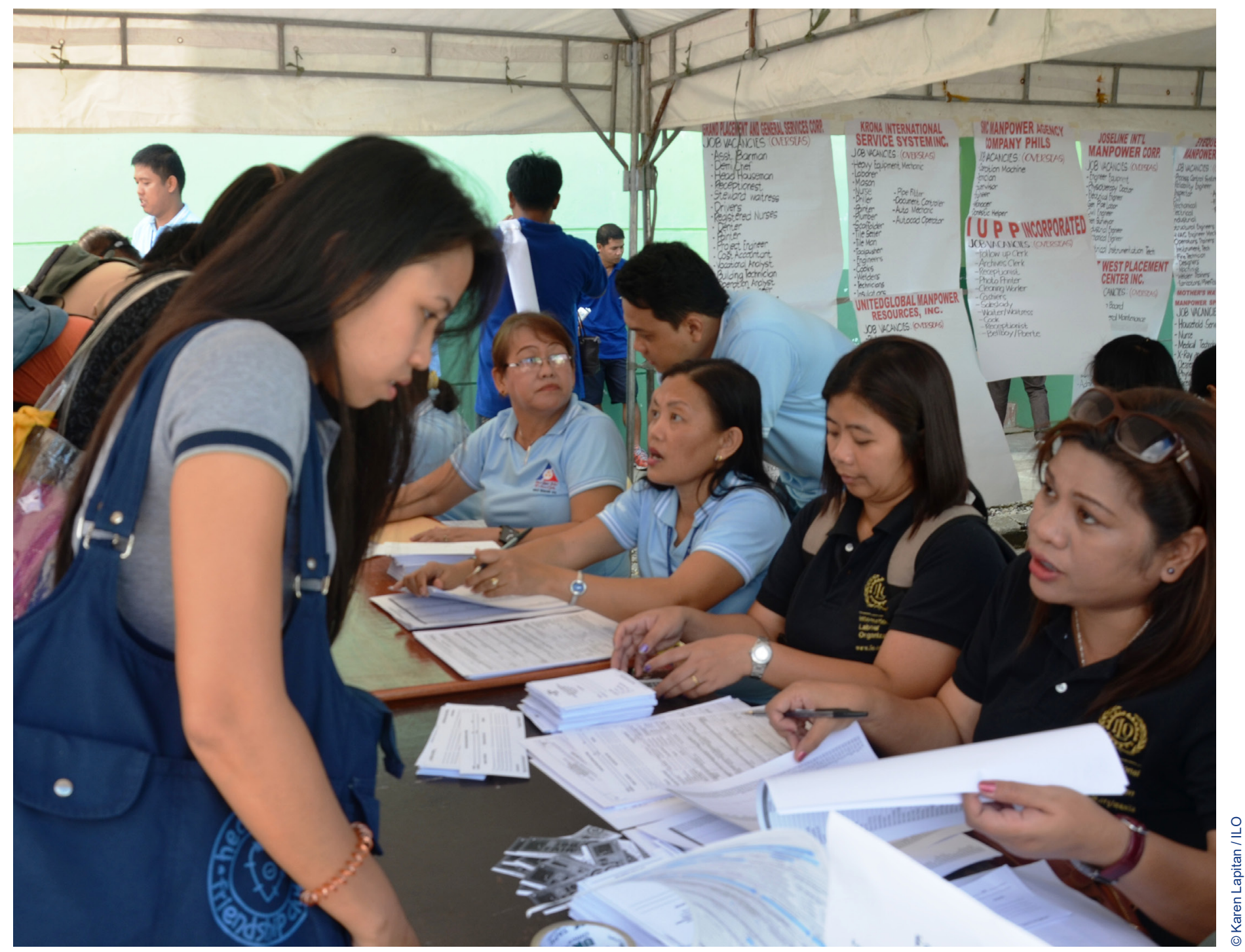

\section{About this impact evaluation}

This brief is based on an impact evaluation report by Emily Beam, Leigh Linden, Stella Quimbo and Heather Richmond, Impact evaluation of the Philippine Special Program for Employment of Students, published in 2018.
This study was funded as part of 3ie's country evidence programme in the Philippines, supported by the Department of Foreign Affairs and Trade, Government of Australia through its embassy in Manila, Philippines.

The International Initiative for Impact Evaluation (3ie) is an international grant-making NGO promoting evidenceinformed development policies and programmes. We are the global leader in funding, producing and synthesising high-quality evidence of what works, for whom, how, why and at what cost. We believe that using better and policy-relevant evidence helps to make development more effective and improve people's lives.

For more information on 3ie's Impact evaluation, contact info@3ieimpact.org or visit our website.

\#3ieimpact.org

@3ieNews f

/3ieimpact
October 2020

$\square$ /3ievideos 